\title{
Progress in diffuse intrinsic pontine glioma: advocating for stereotactic biopsy in the standard of care
}

\author{
John R. Williams, MD,, Christopher C. Young, MD, PhD, ${ }^{1}$ Nicholas A. Vitanza, MD, ${ }^{2}$ \\ Margaret McGrath, MD, ${ }^{1}$ Abdullah H. Feroze, MD, ${ }^{1}$ Samuel R. Browd, MD, PhD, ${ }^{3}$ and \\ Jason S. Hauptman, MD, $\mathrm{PhD}^{3}$
}

1Department of Neurological Surgery, University of Washington; ${ }^{2}$ Division of Hematology/Oncology, Department of Pediatrics, Seattle Children's Hospital; and ${ }^{3}$ Division of Neurosurgery, Seattle Children's Hospital, Seattle, Washington

Diffuse intrinsic pontine glioma (DIPG) is a universally fatal pediatric brainstem tumor affecting approximately 300 children in the US annually. Median survival is less than 1 year, and radiation therapy has been the mainstay of treatment for decades. Recent advances in the biological understanding of the disease have identified the H3K27M mutation in nearly $80 \%$ of DIPGs, leading to the 2016 WHO classification of diffuse midline glioma H3K27M-mutant, a grade IV brainstem tumor. Developments in epigenetic targeting of transcriptional tendencies have yielded potential molecular targets for clinical trials. Chimeric antigen receptor T cell therapy has also shown preclinical promise. Recent clinical studies, including prospective trials, have demonstrated the safety and feasibility of pediatric brainstem biopsy in the setting of DIPG and other brainstem tumors. Given developments in the ability to analyze DIPG tumor tissue to deepen biological understanding of this disease and develop new therapies for treatment, together with the increased safety of stereotactic brainstem biopsy, the authors present a case for offering biopsy to all children with suspected DIPG. They also present their standard operative techniques for image-guided, frameless stereotactic biopsy.

https://thejns.org/doi/abs/10.3171/2019.9.FOCUS19745

KEYWORDS diffuse intrinsic pontine glioma; diffuse midline glioma; biopsy; pediatric cancer; brainstem cancer

$\mathrm{D}$ IFFUSE intrinsic pontine glioma (DIPG) was first described by Wilfred Harris in $1926 .{ }^{25}$ This tumor accounts for nearly $80 \%$ of pediatric brainstem gliomas and is high grade and locally infiltrative with a universally devastating prognosis. ${ }^{17,18,48}$ Histologically, these tumors are astrocytomas ranging from WHO grade II to grade IV, although lower histological grades do not portend a better prognosis. ${ }^{16,68}$ Approximately $10 \%$ of pediatric brain tumors are DIPGs, with about 300 children diagnosed annually in the United States. ${ }^{17}$ Males and females are affected equally, and the median age at diagnosis is 6-7 years old with a median overall survival of 9-11 months. ${ }^{10,36,67}$ Median progression-free survival is 7 months, and DIPG is the leading cause of death from brain tumors in children. ${ }^{10}$ According to a calculation by Vitan$\mathrm{za}$ and Monje based on incidence, median age at diagnosis, and survival, the potential years of life lost annually as a result of this disease are $24,000 .^{68}$

Scientific understanding of the molecular profile of DIPG has increased substantially over the last decade. His- tologically, DIPGs can range from grade II to IV astrocytic glioma changes, but they have always been considered grade IV clinically because of their universally poor prognosis. ${ }^{5,41}$ Landmark studies by Wu, Khuong-Quang, and Schwartzentruber and colleagues, all published in 2012, identified a pathognomonic histone 3 (H3)K27M mutation in nearly $80 \%$ of DIPG cases. ${ }^{32,62,71}$ Further studies showed that the recurrent $\mathrm{H} 3 \mathrm{~K} 27 \mathrm{M}$ mutation found in DIPG is also present in many thalamic and spinal cord gliomas, identifying an "oncohistone" underlying central epigenetic dysregulation. ${ }^{37,63}$ Considered revelatory in terms of the pathophysiological understanding of the disease process, the WHO central nervous system tumor reclassification in 2016 defined a new entity labeled "diffuse midline glioma H3 K27M-mutant" (DMG), which is categorically grade IV. ${ }^{41}$ While the histone mutation in DIPG is now pathognomonic in the new diagnostic term "DMG," there are other molecular aberrations, such those in FGFRI, PDGFR , PI3K, NF1, and NTRK, that are individually infrequent but may each represent molecularly targetable

ABBREVIATIONS CAR = chimeric antigen receptor; DIPG = diffuse intrinsic pontine glioma; DMG = diffuse midline glioma; EGFR = epidermal growth factor receptor. SUBMITTED September 6, 2019. ACCEPTED September 20, 2019.

INCLUDE WHEN CITING DOI: 10.3171/2019.9.FOCUS19745. 


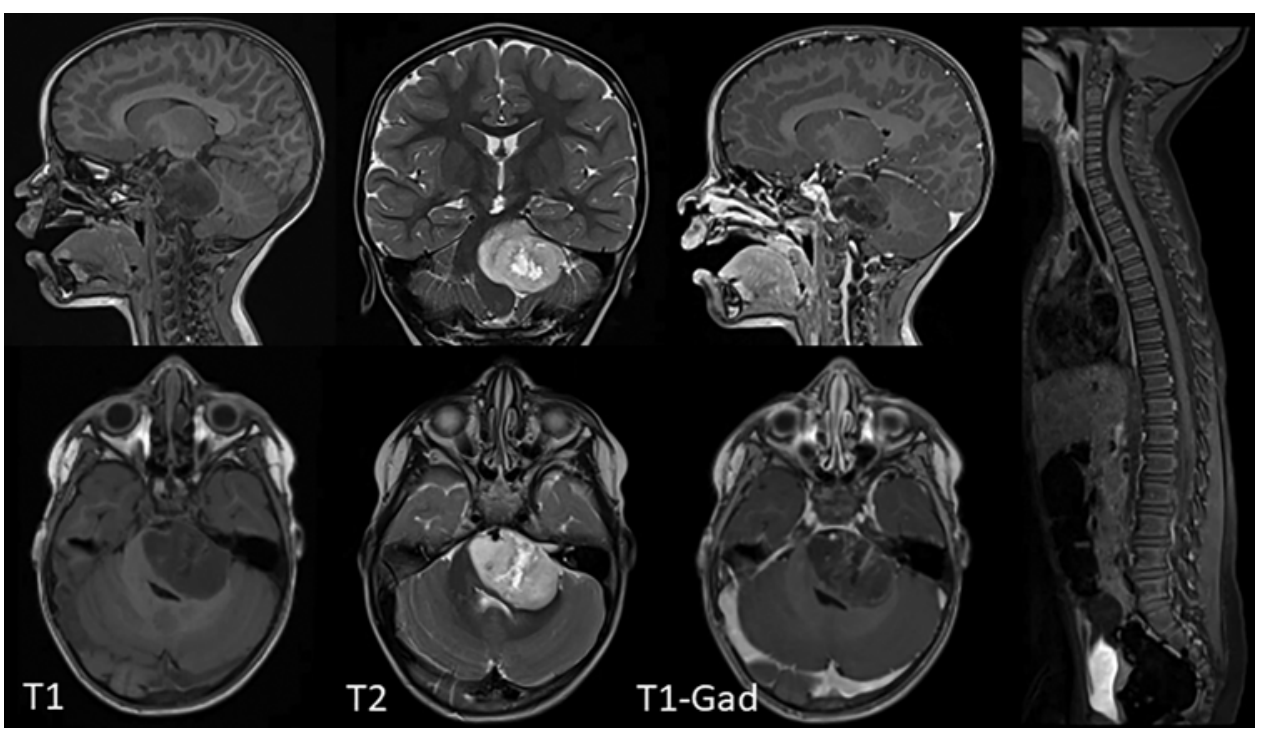

FIG. 1. DIPG is characterized by an expansile pontine-based lesion with varying degrees of neurological signs and symptoms. MRI of the brain with and without gadolinium (Gad) demonstrates a T1 hypointense and a T2 hyperintense lesion with minimal heterogeneous enhancement. The fourth ventricle is effaced, and the basilar artery is encased. MRI of the spine is normal.

subgroups. ${ }^{45}$ While similarly powered proteomic studies are in progress, B7-H3 has already been identified as a potentially targetable surface antigen. ${ }^{46,65}$ Ultimately, nextgeneration molecularly targeted and immunologically targeted trials will rely heavily on biopsy tissue review for target identification and ultimately for appropriate clinical trial enrollment.

For decades, the role of neurosurgery in DIPG has been limited to evaluation and treatment of obstructive hydrocephalus from DIPG and rare biopsies for cases of uncertain diagnosis. ${ }^{1,21}$ Improvements in the understanding of the molecular biology of these tumors have revealed a host of aberrant cell functions and a relative lack of innate immune response. ${ }^{49}$ This knowledge has informed preclinical trials, which in turn have inspired a surge in clinical treatment trials. ${ }^{68}$ Enrollment in many of these trials is contingent on tumor tissue acquisition via biopsy to differentiate among the histone 3 mutations identified to date and to allow for tumor sequencing for individualized treatment pathways based on other relevant mutations and expression dysregulation, such as neurofibromin, neurotrophic receptor tyrosine kinase (NTRK), $O$-6-methylguanine-DNA methyltransferase (MGMT) promoter methylation, epidermal growth factor receptor (EGFR) overexpression, and loss of phosphatase and tensin homolog (PTEN) expression. ${ }^{51,61,70}$ Identifying targetable surface antigens via biopsy-derived tissue has become critical in enrollment in immune-based therapy trials. $^{52,54,55}$ Improvements in the safety and feasibility of brainstem biopsy have occurred coincident with our improved understanding of DIPG biology. ${ }^{22,56}$ Here we present a case for stereotactic brainstem biopsy in all cases of newly diagnosed DIPG as a means to better understand the disease and also as a way to characterize individual tumors based on actionable molecular pathology in order to direct individualized treatment. Ultimately, the goal is to improve survival and quality of life in patients suffering from this devastating disease.

\section{Presentation and Diagnosis}

Children with DIPG tend to present acutely with a median of 1 month of symptoms. ${ }^{67}$ The classic triad of symptoms includes ataxia, pyramidal tract dysfunction, and cranial nerve $(\mathrm{CN})$ palsy, with the abducens nerve $(\mathrm{CN}$ $\mathrm{VI}$ ) being the most commonly affected and usually the first to be affected. ${ }^{16}$

Magnetic resonance imaging of the brain with and without contrast is considered diagnostic for DIPG, though molecular characterization is needed to make the diagnosis of DMG and its subvariants (Fig. 1). ${ }^{1}$ Characteristic features include an expanded pons encasing the basilar artery, increased T2 FLAIR signal in 50\% or more of the ventral pons, and, classically, no T1 contrast enhancement, although it is not unusual to observe small areas of enhancement when necrosis is present. ${ }^{68}$ One study demonstrated that apparent diffusion coefficient values above 1300 correlated positively with improved survival. ${ }^{40}$ Contiguous and distant dissemination from the pons is frequent, and a postmortem review identified not only local infiltration into structures such as the medulla, thalamus, and midbrain, but also distant disease in structures such as the frontal lobe.? Spinal cord disease has been documented at diagnosis, and some authors recommend MRI of the entire spine with and without contrast, with consideration of lumbar puncture in children without hydrocephalus for additional staging information and even the potential for analysis of circulating tumor DNA. ${ }^{27,68}$

While historically the diagnosis of DIPG was made with imaging alone, more recently tissue acquisition has developed an increasingly critical role. Institutional and commercial DNA sequencing have allowed for broader iden- 
tification of the K27M mutation in the histone 3.3 (H3.3) gene $H 3 F 3 A$, the histone 3.2 (H3.2) gene HIST2H3C, and histone 3.1 (H3.1) genes HISTIH3B/C. . $^{32,62,71}$ Immunochemistry is the most cost-efficient test for H3K27M mutations but cannot distinguish among them, and mutation variants have proven clinically important. For instance, the H3.1 variant is specific to pontine gliomas and occurs with higher frequency in females and at a younger age. ${ }^{45}$ Patients with mutations in K27M H3.3 (H3F3A) have been found to be less responsive to radiotherapy, relapse earlier, and have a higher rate of metastatic recurrence as compared to those in the H3.1 $(H I S T 1 H 3 B / C)$ group. ${ }^{8}$ Identification of the H3K27M mutation variant is important in generating the most accurate prognosis and in identifying patients who may qualify for early phase trials targeting specific molecular subgroups.

\section{Current Treatment}

Once the DIPG diagnosis has been established with MRI, multiple services should be engaged for evaluation and possible treatment. Typically, pediatric oncology coordinates most care throughout the course of the disease. Neurosurgical consultation should be initiated in all cases for the management of hydrocephalus when present and for consideration of stereotactic needle biopsy. Although most children do not present with hydrocephalus, some have necrotic centers and/or rapidly progressive disease requiring CSF diversion, preferably with endoscopic third ventriculostomy, and/or emergent radiation therapy. ${ }^{21}$ Even when emergent radiation is not required, radiation oncology should be consulted at the time of diagnosis for expedient therapy planning and early family counseling regarding treatment requirements and potential adverse effects. While often overlooked, early consultation with pediatric palliative care provides a diverse set of support strategies for patients and broader family care networks in the face of this devastating diagnosis. Furthermore, tandem care from palliative and neurooncology teams has been shown to maximize function during treatment and identify signs of disease progression ahead of standard clinical and radiographic assessment. ${ }^{47}$

Dexamethasone is typically prescribed at the time of diagnosis for alleviation and stabilization of related neurological symptoms. While high-dose steroids offer early relief, their well-established side effect profile, including impaired sleep, wound healing, behavior, and endocrine and metabolic functional effects, limits their long-term utility. ${ }^{11}$ Additionally, corticosteroids reinforce the blood-brain barrier, limiting penetration by extant and experimental systemic therapies, and may shorten survival..$^{15,28,44,57}$ For these reasons, dexamethasone use should be limited to short bursts and weaned as quickly as tolerable.

Focal radiotherapy is the only intervention with substantial evidence supporting increased overall survival in pediatric DIPG. In its absence, survival is approximately 5 months, and overall survival increases $2-4$ months with standard treatment. ${ }^{34}$ Treatment typically consists of conformational photon radiotherapy directly to the tumor for a total of 54 Gy in daily 1.8-Gy increments over 6 weeks. Similar outcomes have been demonstrated with a hypo- fractionated regimen. ${ }^{31,72}$ Reirradiation is considered with disease progression and has been demonstrated to be safe, with a small survival benefit and a positive effect on symptoms..$^{30,35}$ No radiosensitizing agents have shown survival benefit to date. ${ }^{2,4}$

Further, hundreds of trials of cytotoxic and myeloablative chemotherapy have shown no survival benefit in this disease. ${ }^{60}$ Intensive chemotherapeutic treatment regimens with myeloablative dosing requiring stem cell transplant, gemcitabine, capecitabine, several tyrosine kinase inhibitors, and a monoclonal EGFR antibody have been tested in the setting of DIPG without improvement in overall or progression-free survival. ${ }^{49} \mathrm{~A}$ phase II trial using the EGFR inhibitor gefitinib in combination with radiation therapy did show overall survival rates that were "nominally superior" to those in contemporaneous trial cohorts. ${ }^{58}$ Curiously, single-agent temozolomide, which has repeatedly shown some efficacy in adult high-grade gliomas, does not alter DIPG outcomes in conventional or metronomic dosing. ${ }^{9,29,64}$ This wide resistance to conventional chemoradiation treatments has heightened the urgency directed toward understanding the molecular biology of DIPG in the pursuit of targeted therapies for this lethal disease.

\section{Research Progress}

Increased tissue acquisition has allowed for improved understanding of the molecular biology of DIPG and its increasing number of known variants. Expanding awareness of DIPG as a neurosurgical disease has also opened the door to new prospects in individualized medicine and targeted trials.

Preclinical drug screens have shown particular promise in epigenetic targeted agents, leading to clinical trials to evaluate the histone deacetylase (HDAC) inhibitor panobinostat. ${ }^{20}$ Epigenetic studies have also identified oncogenic transcription targets including CDK7 blockade and BRD4 inhibition. ${ }^{53}$ Antitumor activity has been demonstrated by inhibiting K27 demethylase JMJD3 via GSKJ4, showing promise in targeting defective transcription mechanisms in DIPG. ${ }^{26}$ Interestingly, DIPG demonstrates an important distinction from adult glioblastoma (GBM) in its microenvironment, exhibiting comparatively low levels of immunosuppression and inflammation. ${ }^{38,39}$ This lack of immunosuppression makes DIPG more susceptible to chimeric antigen receptor (CAR) T cell therapy. Preclinical in vivo studies targeting the heavily expressed disialoganglioside GD2 nearly eradicated H2K27M-mutant DMG tumors in xenograft mouse models, prompting study of additional targets such as B7-H3.46,50 This work provided the foundation for the planned clinical trial of GD2-specific CAR T cell therapy for DIPG. Current CAR $\mathrm{T}$ cell trials in place are deploying HER2-specific cells (BrainChild-01, NCT03500991) and EGFR806-specific cells (BrainChild-02, NCT03638167) in children with refractory or recurrent central nervous system tumors. Both BrainChild-01 and BrainChild-02 have excluded DIPG, but their evaluation of locoregional delivery of CAR T cells will provide critical information regarding the inflammatory response as the design for BrainChild-03, a 
clinical trial of B7-H3-specific CAR T cells for treatment of DIPG, is finalized. ${ }^{68}$

Biopsy-dependent tissue has also allowed tumor sequencing for individualized treatment pathways based on other relevant mutations and expression dysregulation identified in DIPG, including neurofibromin, NTRK, MGMT promoter methylation, EGFR overexpression, and loss of PTEN expression. ${ }^{49,51,61,70}$ Tumor cell epitope identification after biopsy has also inspired immunotherapy trials using vaccine-based targeting. ${ }^{52}$ Biopsy has also been incorporated into published standard of care protocols and is being used to determine tumor penetration by drugs administered systemically. ${ }^{19}$

Progress is also being realized in drug delivery mechanisms. Convection-enhanced delivery (CED) is the practice of stereotactically navigating a drug delivery catheter into the pontine tumor directly. Theorized advantages to this technique are twofold. First, the catheter can overcome the blood-brain barrier and deliver a greater variety of drugs to the tumor directly. Second, the drug of interest is delivered with a positive pressure pump, allowing for broader distribution than simple diffusion. ${ }^{23}$ CED has been tested in treating adult GBM, and in a phase I trial by Souweidane and colleagues, the technique has been demonstrated to be safe and effective in terms of local delivery without systemic toxicity or serious operative complication. ${ }^{65}$

\section{Role of Neurosurgery in DIPG Treatment and Biopsy}

Thus, a window into the biological understanding of DIPG has opened. Patient-derived cells, animal models, and genetic engineering are paving new roads toward meaningful treatment. ${ }^{3,43,49,66}$ In 1993, Leland Albright presented a convincing case against all operative intervention for DIPG given the diagnostic capacity of MRI, unacceptable morbidity of resection, and lack of utility of tissue biopsy. ${ }^{1}$ However, in the intervening decades, the increased safety and feasibility of stereotactic biopsy, together with research developments of clinical significance, command reexamination of this stance. Current data show that stereotactic biopsy can be performed safely with minimal morbidity and mortality and is associated with a high pathological and molecular diagnostic yield, making a strong case for carefully executed biopsy in all cases of suspected DIPG at institutions with the capacity for the intervention.

\section{Feasibility, Safety, and Utility of Brainstem Biopsies}

Several prospective and retrospective studies, as well as meta-analyses, have demonstrated the relative safety of stereotactic biopsy procedures in multiple centers worldwide. In the DIPG Biology and Treatment Study, ${ }^{22}$ a national clinical trial for the treatment of DIPG, the feasibility and safety of brainstem biopsy were demonstrated in 50 patients from 23 institutions. Using a frameless, stereotactic transcerebellar approach, researchers obtained diagnostic tissue in 48/50 patients (96\%). No significant hemorrhagic complication was reported. One patient experienced permanent hemiparesis, and there was no procedure-related mortality. Similarly, in a national pediatric brain tumor registry study in Germany, brainstem biopsy was shown to be safe. ${ }^{56}$ In the Individualised Therapy For Relapsed Malignancies in Childhood (INFORM) registry, 21 patients underwent brainstem biopsy at 12 centers over a 3-year period. Notably, 12 of the 21 patients underwent frame-based biopsy, while 5 patients underwent open surgical biopsy. Nevertheless, sufficient tissue was obtained in all patients for pathological and molecular diagnosis. One patient developed hydrocephalus requiring shunting, and another patient experienced permanent neurological deficit. The largest pediatric biopsy series consists of 130 patients from a single French institution. ${ }^{59}$ Patients in that study underwent Leksell frame-based, stereotactic transcerebellar biopsy. Every biopsy in that series was diagnostic for DIPG, and there were 5 cases of transient worsening of neurological deficit, which either resolved or significantly improved without mortality. An earlier meta-analysis of 38 studies with 1480 adult and pediatric patients showed a $96.2 \%$ diagnostic success rate associated with $7.8 \%$ morbidity (1.7\% permanent) and $0.9 \%$ mortality. ${ }^{33}$ Another meta-analysis of 18 studies with 735 pediatric brainstem biopsies suggested that diagnostic success was similarly high at $96.1 \%$ with $6.7 \%$ morbidity $(0.6 \%$ permanent) and $0.6 \%$ mortality. ${ }^{24}$ Cumulatively, there is a body of evidence suggesting that brainstem biopsy can be safely performed with a high diagnostic yield. Furthermore, meta-analyses suggest that different surgical approaches such as transfrontal versus transcerebellar, ${ }^{6,13,33}$ the use of frame-based versus frameless stereotactic biopsy, ${ }^{14,69}$ or the use of robot-assisted systems ${ }^{6,12}$ have all consistently resulted in an excellent diagnostic yield with relatively minor morbidity. Biopsy tissue has revitalized the study of DIPG, leading to new classifications, dozens of promising preclinical trials, individualized therapy targeting patientspecific mutations, and multiple clinical trials.

\section{Operative Preparation and Surgical Approach}

At our institution, established standards of care are initiated upon diagnosis, including the administration of a short course of high-dose steroids, treatment of hydrocephalus if present, and team consultation and family counseling. The patients' families are universally offered stereotactic biopsy and counseled on the risks and benefits of the procedure based on the information described here.

We use two systems for the procedure: a Vertek biopsy device (Medtronic; Fig. 2A and B) and a robot-assisted approach (ROSA robotic system, Zimmer-Biomet USA). Routine CT and MRI data for navigation are acquired. Anatomy is carefully reviewed to plan the biopsy trajectory, avoiding eloquent areas, vascular structures, and transependymal breach. Cystic or necrotic components of the tumor are avoided wherever possible to increase the diagnostic yield. Although both transfrontal and transcerebellar approaches to the brainstem are reported to have similar rates of diagnostic success, as well as morbidity and mortality, ${ }^{13,33}$ we prefer the suboccipital transcerebellar approach, lateral to the midline, taking the shortest 

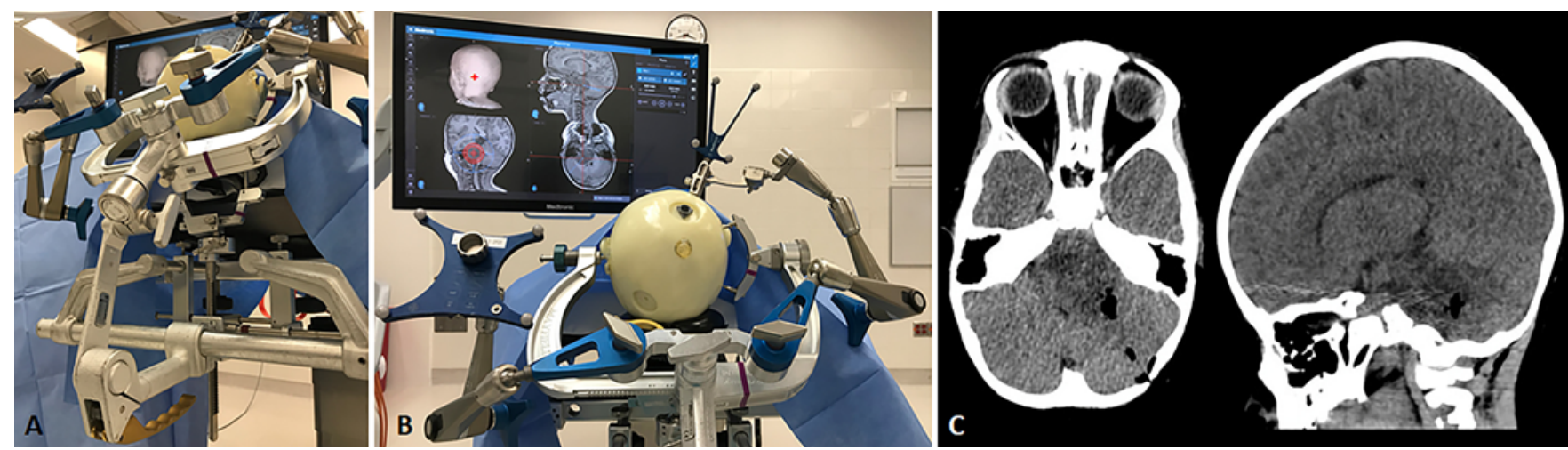

FIG. 2. Frameless stereotactic transcerebellar biopsy. A: The pediatric patient is positioned prone on the operating table. The head is immobilized with the Mayfield ${ }^{\circledR}$ skull clamp with an 18 -lb torque screw and supported by the Mayfield ${ }^{\circledR}$ Infinity Support System. B: The Stealth frameless navigation system is registered with high-resolution CT with fiducial markers and surface tracing. Merged MRI of the brain is used to plan the biopsy trajectory, taking care to avoid vascular structures, eloquent areas, and ependymal transgression. The Vertek ${ }^{\circledR}$ articulating arm is used to manually navigate the biopsy needle to the entry point and then to the biopsy target. C: Location of the biopsy and the absence of immediate surgical complication are confirmed on postoperative CT.

route to the lesion, generally by transgressing the middle cerebellar peduncle. ${ }^{22}$ The location of the venous sinuses, deep cerebellar nuclei, and ventral pontine motor tracts is noted and meticulously avoided. The use of diffusion tensor imaging (DTI) to visualize white matter tracts and plan the biopsy trajectory has been reported:42 however, the density of white matter tracts through this region makes integrating DTI information into trajectory planning extremely challenging.

Standard preoperative care, anesthesia, sterile preparation, and positioning are utilized. Mannitol and hyperventilation are avoided to prevent volumetric changes, which can distort intracranial anatomy relative to navigation imaging sequences. The patient is positioned prone with the head held in a Mayfield head holder. For very young patients whose head cannot be held by the head holder alone, the Mayfield Infinity Support System (Integra) is used, which supports the face on a horseshoe headrest, and the head is stabilized with only $18 \mathrm{lbs}$ of torque (Fig. 2A). In order to achieve precision of $1 \mathrm{~mm}$ or less when using the Vertek frameless system, we frequently use fiducial markers in combination with skin tracing for registration with the StealthStation S8 system (Medtronic). When the ROSA robot is employed, the Mayfield head holder is docked to the robot station. Planning, similar to what is performed on the StealthStation, is done within the ROSA software platform. In our registration, we prefer to place five bone fiducials along the midline and slightly lateral to midline for registration. This is followed by intraoperative CT scanning using the O-arm (Medtronic), and the results are fused with the preoperative MRI scan in the ROSA software. Standard procedural techniques are used for opening, tissue acquisition, closing, and hemostasis. We ensure our neuropathology colleagues are on call to interpret the first-acquired tissue as frozen preparation to ensure diagnostic tumor tissue is being collected. At the end of the procedure, immediate head CT is performed to confirm the site of biopsy and exclude complications (Fig. 2C).

\section{Conclusions}

DIPG is a high-grade brainstem tumor affecting approximately 300 children annually in the United States. It is a devastating diagnosis with a prognosis of death within a year for most affected children. Historically, care has focused on radiotherapy and palliative measures as these lesions are anatomically unresectable and have not responded to conventional chemotherapy. Biopsy, once reserved only for cases of questionable diagnosis, has reemerged as a routine consideration in DIPG diagnosis, as advances in our molecular understanding of the disease have led to a number of new subclassifications with clinically relevant prognostic differences. More importantly, the availability of tumor tissue has driven the development of trials, which have shown promise in tumor control in experimental models. Those models have been the foundation of a number of active clinical trials identifying and treating new targets in DIPG. At the same time, recent studies of brainstem biopsy using improved imaging and stereotactic navigation technology have shown improved safety and feasibility of the procedure. While radiotherapy remains the only meaningful intervention in terms of overall survival, tissue sequencing and analysis has increased optimism in the field that treatment breakthroughs may be forthcoming. For these reasons, we advocate for an informed discussion with patients and families about brainstem biopsy as part of the evolving standard of care in the treatment of DIPG.

\section{Acknowledgments}

We wish to thank Carrie Waring, RN, BSN, for her assistance with preparation of the figures, and Sharon Durfy, $\mathrm{PhD}$, for her assistance with manuscript preparation.

\section{References}

1. Albright AL, Packer RJ, Zimmerman R, Rorke LB, Boyett $\mathrm{J}$, Hammond GD: Magnetic resonance scans should replace 
biopsies for the diagnosis of diffuse brain stem gliomas: a report from the Children's Cancer Group. Neurosurgery 33:1026-1030, 1993

2. Aquino-Parsons C, Hukin J, Green A: Concurrent carbogen and radiation therapy in children with high-risk brainstem gliomas. Pediatr Blood Cancer 50:397-399, 2008

3. Biery M, Myers C, Girard E, Morris S, Carmack S, Noll A, et al: DIPG-35. A novel HDAC inhibitor in new patient-derived diffuse intrinsic pontine glioma (DIPG) models. Neuro Oncol 20 (Suppl 2):i56, 2018 (Abstract)

4. Bradley KA, Zhou T, McNall-Knapp RY, Jakacki RI, Levy AS, Vezina G, et al: Motexafin-gadolinium and involved field radiation therapy for intrinsic pontine glioma of childhood: a children's oncology group phase 2 study. Int J Radiat Oncol Biol Phys 85:e55-e60, 2013

5. Buczkowicz P, Bartels U, Bouffet E, Becher O, Hawkins C: Histopathological spectrum of paediatric diffuse intrinsic pontine glioma: diagnostic and therapeutic implications. Acta Neuropathol 128:573-581, 2014

6. Carai A, Mastronuzzi A, De Benedictis A, Messina R, Cacchione A, Miele E, et al: Robot-assisted stereotactic biopsy of diffuse intrinsic pontine glioma: a single-center experience. World Neurosurg 101:584-588, 2017

7. Caretti V, Bugiani M, Freret M, Schellen P, Jansen M, van Vuurden D, et al: Subventricular spread of diffuse intrinsic pontine glioma. Acta Neuropathol 128:605-607, 2014

8. Castel D, Philippe C, Calmon R, Le Dret L, Truffaux N, Boddaert N, et al: Histone H3F3A and HIST1H3B K27M mutations define two subgroups of diffuse intrinsic pontine gliomas with different prognosis and phenotypes. Acta Neuropathol 130:815-827, 2015

9. Cohen KJ, Heideman RL, Zhou T, Holmes EJ, Lavey RS, Bouffet E, et al: Temozolomide in the treatment of children with newly diagnosed diffuse intrinsic pontine gliomas: a report from the Children's Oncology Group. Neuro Oncol 13:410-416, 2011

10. Cooney T, Lane A, Bartels U, Bouffet E, Goldman S, Leary SES, et al: Contemporary survival endpoints: an International Diffuse Intrinsic Pontine Glioma Registry study. Neuro Oncol 19:1279-1280, 2017

11. Curtis JR, Westfall AO, Allison J, Bijlsma JW, Freeman A, George V, et al: Population-based assessment of adverse events associated with long-term glucocorticoid use. Arthritis Rheum 55:420-426, 2006

12. De Benedictis A, Trezza A, Carai A, Genovese E, Procaccini E, Messina R, et al: Robot-assisted procedures in pediatric neurosurgery. Neurosurg Focus 42(5):E7, 2017

13. Dellaretti M, Reyns N, Touzet G, Dubois F, Gusmão S, Pereira JLB, et al: Stereotactic biopsy for brainstem tumors: comparison of transcerebellar with transfrontal approach. Stereotact Funct Neurosurg 90:79-83, 2012

14. Dhawan S, He Y, Bartek J Jr, Alattar AA, Chen CC: Comparison of frame-based versus frameless intracranial stereotactic biopsy: systematic review and meta-analysis. World Neurosurg 127:607-616.e4, 2019

15. Fauquette W, Amourette C, Dehouck MP, Diserbo M: Radiation-induced blood-brain barrier damages: an in vitro study. Brain Res 1433:114-126, 2012

16. Fisher PG, Breiter SN, Carson BS, Wharam MD, Williams JA, Weingart JD, et al: A clinicopathologic reappraisal of brain stem tumor classification. Identification of pilocystic astrocytoma and fibrillary astrocytoma as distinct entities. Cancer 89:1569-1576, 2000

17. Freeman CR, Farmer JP: Pediatric brain stem gliomas: a review. Int J Radiat Oncol Biol Phys 40:265-271, 1998

18. Gelder CL, Hawkins C, Zapotocky M, Dirks P, Bartels U, Bouffet E: Diffuse intrinsic pontine glioma ventricular peritoneal shunt metastasis: a case report and literature review. Childs Nerv Syst 35:861-864, 2019
19. Green A: Gemcitabine in newly-diagnosed diffuse intrinsic pontine glioma. ClinicalTrials.gov (https://clinicaltrials.gov/ ct2/show/NCT02992015) [Accessed November 6, 2019]

20. Grasso CS, Tang Y, Truffaux N, Berlow NE, Liu L, Debily MA, et al: Functionally defined therapeutic targets in diffuse intrinsic pontine glioma. Nat Med 21:555-559, 2015

21. Guida L, Roux FE, Massimino M, Marras CE, Sganzerla E, Giussani C: Safety and efficacy of endoscopic third ventriculostomy in diffuse intrinsic pontine glioma related hydrocephalus: a systematic review. World Neurosurg 124:29-35, 2018

22. Gupta N, Goumnerova LC, Manley P, Chi SN, Neuberg $\mathrm{D}$, Puligandla M, et al: Prospective feasibility and safety assessment of surgical biopsy for patients with newly diagnosed diffuse intrinsic pontine glioma. Neuro Oncol 20:1547-1555, 2018

23. Halle B, Mongelard K, Poulsen FR: Convection-enhanced drug delivery for glioblastoma: a systematic review focused on methodological differences in the use of the convectionenhanced delivery method. Asian J Neurosurg 14:5-14, 2019

24. Hamisch C, Kickingereder P, Fischer M, Simon T, Ruge MI: Update on the diagnostic value and safety of stereotactic biopsy for pediatric brainstem tumors: a systematic review and meta-analysis of 735 cases. J Neurosurg Pediatr 20:261-268, 2017

25. Harris W: A case of pontine glioma, with special reference to the paths of gustatory sensation. Proc R Soc Med 19 (Neurol Sect): 1-5, 1926

26. Hashizume R, Andor N, Ihara Y, Lerner R, Gan H, Chen X, et al: Pharmacologic inhibition of histone demethylation as a therapy for pediatric brainstem glioma. Nat Med 20:13941396, 2014

27. Huang TY, Piunti A, Lulla RR, Qi J, Horbinski CM, Tomita $\mathrm{T}$, et al: Detection of histone H3 mutations in cerebrospinal fluid-derived tumor DNA from children with diffuse midline glioma. Acta Neuropathol Commun 5:28, 2017

28. Hue CD, Cho FS, Cao S, Dale Bass CR, Meaney DF, Morrison B III: Dexamethasone potentiates in vitro blood-brain barrier recovery after primary blast injury by glucocorticoid receptor-mediated upregulation of ZO-1 tight junction protein. J Cereb Blood Flow Metab 35:1191-1198, 2015

29. Jalali R, Raut N, Arora B, Gupta T, Dutta D, Munshi A, et al: Prospective evaluation of radiotherapy with concurrent and adjuvant temozolomide in children with newly diagnosed diffuse intrinsic pontine glioma. Int J Radiat Oncol Biol Phys 77:113-118, 2010

30. Janssens GO, Gandola L, Bolle S, Mandeville H, RamosAlbiac M, van Beek K, et al: Survival benefit for patients with diffuse intrinsic pontine glioma (DIPG) undergoing re-irradiation at first progression: a matched-cohort analysis on behalf of the SIOP-E-HGG/DIPG working group. Eur J Cancer 73:38-47, 2017

31. Janssens GO, Jansen MH, Lauwers SJ, Nowak PJ, Oldenburger FR, Bouffet E, et al: Hypofractionation vs conventional radiation therapy for newly diagnosed diffuse intrinsic pontine glioma: a matched-cohort analysis. Int J Radiat Oncol Biol Phys 85:315-320, 2013

32. Khuong-Quang DA, Buczkowicz P, Rakopoulos P, Liu XY, Fontebasso AM, Bouffet E, et al: K27M mutation in histone H3.3 defines clinically and biologically distinct subgroups of pediatric diffuse intrinsic pontine gliomas. Acta Neuropathol 124:439-447, 2012

33. Kickingereder P, Willeit P, Simon T, Ruge MI: Diagnostic value and safety of stereotactic biopsy for brainstem tumors: a systematic review and meta-analysis of 1480 cases. Neurosurgery 72:873-882, 2013

34. Langmoen IA, Lundar T, Storm-Mathisen I, Lie SO, Hovind 
KH: Management of pediatric pontine gliomas. Childs Nerv Syst 7:13-15, 1991

35. Lassaletta A, Strother D, Laperriere N, Hukin J, Vanan MI, Goddard K, et al: Reirradiation in patients with diffuse intrinsic pontine gliomas: the Canadian experience. Pediatr Blood Cancer 65:e26988, 2018

36. Lassman LP, Arjona VE: Pontine gliomas of childhood. Lancet 1:913-915, 1967

37. Lewis PW, Müller MM, Koletsky MS, Cordero F, Lin S, Banaszynski LA, et al: Inhibition of PRC2 activity by a gainof-function $\mathrm{H} 3$ mutation found in pediatric glioblastoma. Science 340:857-861, 2013

38. Lieberman NAP, DeGolier K, Kovar HM, Davis A, Hoglund V, Stevens J, et al: Characterization of the immune microenvironment of diffuse intrinsic pontine glioma: implications for development of immunotherapy. Neuro Oncol 21:83-94, 2019

39. Lin GL, Nagaraja S, Filbin MG, Suvà ML, Vogel H, Monje $\mathrm{M}$ : Non-inflammatory tumor microenvironment of diffuse intrinsic pontine glioma. Acta Neuropathol Commun 6:51, 2018

40. Lober RM, Cho YJ, Tang Y, Barnes PD, Edwards MS, Vogel $\mathrm{H}$, et al: Diffusion-weighted MRI derived apparent diffusion coefficient identifies prognostically distinct subgroups of pediatric diffuse intrinsic pontine glioma. J Neurooncol 117:175-182, 2014

41. Louis DN, Perry A, Reifenberger G, von Deimling A, Figarella-Branger D, Cavenee WK, et al: The 2016 World Health Organization Classification of Tumors of the Central Nervous System: a summary. Acta Neuropathol 131:803820, 2016

42. Low SYY, Soh SY, Chen MW, Ng LP, Low DCY, Seow WT: DTI fusion with conventional MR imaging in intra-operative MRI suite for paediatric brainstem glioma biopsy. Childs Nerv Syst 34:19-21, 2018

43. Lowe BR, Maxham LA, Hamey JJ, Wilkins MR, Partridge JF: Histone H3 mutations: an updated view of their role in chromatin deregulation and cancer. Cancers (Basel) 11:E660, 2019

44. Luedi MM, Singh SK, Mosley JC, Hatami M, Gumin J, Sulman EP, et al: A dexamethasone-regulated gene signature is prognostic for poor survival in glioblastoma patients. J Neurosurg Anesthesiol 29:46-58, 2017

45. Mackay A, Burford A, Carvalho D, Izquierdo E, Fazal-Salom J, Taylor KR, et al: Integrated molecular meta-analysis of 1,000 pediatric high-grade and diffuse intrinsic pontine glioma. Cancer Cell 32:520-537.e5, 2017

46. Majzner RG, Theruvath JL, Nellan A, Heitzeneder S, Cui Y, Mount CW, et al: CAR T cells targeting B7-H3, a pan-cancer antigen, demonstrate potent preclinical activity against pediatric solid tumors and brain tumors. Clin Cancer Res 25:2560-2574, 2019

47. Mandrell BN, Baker J, Levine D, Gattuso J, West N, Sykes A, et al: Children with minimal chance for cure: parent proxy of the child's health-related quality of life and the effect on parental physical and mental health during treatment. J Neurooncol 129:373-381, 2016

48. Marcus KJ, Karajannis MA: Diffuse intrinsic pontine glioma. UpToDate (https://www.uptodate.com/contents/ diffuse-intrinsic-pontine-glioma) [Accessed November 6, 2019]

49. Mathew RK, Rutka JT: Diffuse intrinsic pontine glioma: clinical features, molecular genetics, and novel targeted therapeutics. J Korean Neurosurg Soc 61:343-351, 2018

50. Mount CW, Majzner RG, Sundaresh S, Arnold EP, Kadapakkam M, Haile S, et al: Potent antitumor efficacy of anti-GD2 CAR T cells in H3-K27M+ ${ }^{+}$diffuse midline gliomas. Nat Med 24:572-579, 2018

51. Mueller S: Molecular profiling for individualized treatment plan for DIPG. ClinicalTrials.gov (https://clinicaltrials.gov/ ct2/show/NCT02274987) [Accessed November 6, 2019]

52. Mueller S, Okada H: H3.3K27M peptide vaccine for children with newly diagnosed DIPG and other gliomas. ClinicalTrials.gov (https://clinicaltrials.gov/ct2/show/ NCT02960230) [Accessed November 6, 2019]

53. Nagaraja S, Vitanza NA, Woo PJ, Taylor KR, Liu F, Zhang $\mathrm{L}$, et al: Transcriptional dependencies in diffuse intrinsic pontine glioma. Cancer Cell 31:635-652.e6, 2017

54. Park J: EGFR806-specific CAR T cell locoregional immunotherapy for EGFR-positive recurrent or refractory pediatric CNS tumors. ClinicalTrials.gov (https:// clinicaltrials.gov/ct2/show/NCT03638167) [Accessed November 6, 2019]

55. Park J: HER2-specific CAR T cell locoregional immunotherapy for HER2-positive recurrent/refractory pediatric CNS tumors. ClinicalTrials.gov (https:// clinicaltrials.gov/ct2/show/NCT03500991) [Accessed November 6, 2019]

56. Pfaff E, El Damaty A, Balasubramanian GP, BlattnerJohnson M, Worst BC, Stark S, et al: Brainstem biopsy in pediatric diffuse intrinsic pontine glioma in the era of precision medicine: the INFORM study experience. Eur J Cancer 114:27-35, 2019

57. Pitter KL, Tamagno I, Alikhanyan K, Hosni-Ahmed A, Pattwell SS, Donnola S, et al: Corticosteroids compromise survival in glioblastoma. Brain 139:1458-1471, 2016

58. Pollack IF, Stewart CF, Kocak M, Poussaint TY, Broniscer A, Banerjee A, et al: A phase II study of gefitinib and irradiation in children with newly diagnosed brainstem gliomas: a report from the Pediatric Brain Tumor Consortium. Neuro Oncol 13:290-297, 2011

59. Puget S, Beccaria K, Blauwblomme T, Roujeau T, James $\mathrm{S}$, Grill J, et al: Biopsy in a series of 130 pediatric diffuse intrinsic pontine gliomas. Childs Nerv Syst 31:1773-1780, 2015

60. Robison NJ, Kieran MW: Diffuse intrinsic pontine glioma: a reassessment. J Neurooncol 119:7-15, 2014

61. Roussy G: Biological medicine for diffuse intrinsic pontine glioma (DIPG) eradication. ClinicalTrials.gov (https:// clinicaltrials.gov/ct2/show/NCT02233049) [Accessed November 6, 2019]

62. Schwartzentruber J, Korshunov A, Liu XY, Jones DTW, Pfaff E, Jacob K, et al: Driver mutations in histone H3.3 and chromatin remodelling genes in paediatric glioblastoma. Nature 482:226-231, 2012

63. Shankar GM, Lelic N, Gill CM, Thorner AR, Van Hummelen $\mathrm{P}, \mathrm{Wis}$ off $\mathrm{JH}$, et al: BRAF alteration status and the histone H3F3A gene K27M mutation segregate spinal cord astrocytoma histology. Acta Neuropathol 131:147-150, 2016

64. Sharp JR, Bouffet E, Stempak D, Gammon J, Stephens D, Johnston DL, et al: A multi-centre Canadian pilot study of metronomic temozolomide combined with radiotherapy for newly diagnosed paediatric brainstem glioma. Eur J Cancer 46:3271-3279, 2010

65. Souweidane MM, Kramer K, Pandit-Taskar N, Zhou Z, Haque S, Zanzonico P, et al: Convection-enhanced delivery for diffuse intrinsic pontine glioma: a single-centre, doseescalation, phase 1 trial. Lancet Oncol 19:1040-1050, 2018

66. Tsoli M, Shen H, Mayoh C, Franshaw L, Ehteda A, Upton D, et al: International experience in the development of patientderived xenograft models of diffuse intrinsic pontine glioma. J Neurooncol 141:253-263, 2019

67. Veldhuijzen van Zanten SE, Jansen MH, Sanchez Aliaga E, van Vuurden DG, Vandertop WP, Kaspers GJ: A twentyyear review of diagnosing and treating children with diffuse intrinsic pontine glioma in The Netherlands. Expert Rev Anticancer Ther 15:157-164, 2015

68. Vitanza NA, Monje M: Diffuse intrinsic pontine glioma: 
from diagnosis to next-generation clinical trials. Curr Treat Options Neurol 21:37, 2019

69. Woodworth GF, McGirt MJ, Samdani A, Garonzik I, Olivi A, Weingart JD: Frameless image-guided stereotactic brain biopsy procedure: diagnostic yield, surgical morbidity, and comparison with the frame-based technique. J Neurosurg 104:233-237, 2006

70. Wright KD: Molecularly determined treatment of diffuse intrinsic pontine gliomas (DIPG). ClinicalTrials.gov (https://clinicaltrials.gov/ct2/show/NCT01182350) [Accessed November 6, 2019]

71. Wu G, Broniscer A, McEachron TA, Lu C, Paugh BS, Becksfort J, et al: Somatic histone $\mathrm{H} 3$ alterations in pediatric diffuse intrinsic pontine gliomas and non-brainstem glioblastomas. Nat Genet 44:251-253, 2012

72. Zaghloul MS, Eldebawy E, Ahmed S, Mousa AG, Amin A, Refaat A, et al: Hypofractionated conformal radiotherapy for pediatric diffuse intrinsic pontine glioma (DIPG): a randomized controlled trial. Radiother Oncol 111:35-40, 2014

\section{Disclosures}

Dr. Hauptman is a consultant for Medtronic.

\section{Author Contributions}

Conception and design: Williams, Young, Vitanza, Browd, Hauptman. Drafting the article: Williams, Young. Critically revising the article: Williams, Young, Vitanza, Hauptman. Reviewed submitted version of manuscript: all authors. Approved the final version of the manuscript on behalf of all authors: Williams. Administrative/technical/material support: Young. Study supervision: Browd, Hauptman. Literature acquisition and analysis: McGrath, Feroze.

\section{Correspondence}

John R. Williams: University of Washington, Seattle, WA. respub@uw.edu. 